\title{
Beitrag zur Kenntnis der Vogeltænien
}

\author{
von \\ O. FUHRMANN \\ Assistent am Vorgleichend-Anatomischen Institut der Universität Genf.
}

Mit Tafel IV

II.

Ueber das Subgenus Davainea

Dav. leptosoma Diesing, Dav. tauricollis Chapm.,

Dav. musculosa nov. spec.

Davainea leptosoma Diesing.

(Fig. 1 u. 2.)

Dieser Cestode kommt im Darme vieler Psittacusarten vor; er besitzt eine Länge von ca. $190 \mathrm{~mm}$. bei einem Maximum der Breite von 2,7 mm. Das Rostrum ist nach KRABBE (1869) bewaffnet mit einem wahrscheinlich doppelten Kranze von 70 Hacken (Fig. 1). Leider war der einzige Scolex der mir zur Verfügung stehenden Exemplare in schlechtem Zustande, so dass ich keine weiteren Angaben über denselben machen kann. Die Glieder der Strobilenkette sind sehr kurz, ihr Längenund Breiten-Verhältnis bleibt überall fast dasselbe und zwar $1: 7$.

Die Körperdecke besteht aus einer starken Cuticula, unter welcher ein gleichmässig feines Netz von Ring- und Längs- 
muskelfasern liegt, auf welches die subcuticularen Zellen folgen.

Die innere Parenchymmuskulatur setzt sich aus Längs- und Transversal-Fasern und aus Dorsoventralmuskeln zusammen. Fast die ganze äussere Parenchymzone ist erfültt von Längsmuskeln die teils einzeln, teils bis zu Bündeln von acht Fasern vereinigt, das Rindenparenchym durchziehen. An den Seiten der Proglottiden ist die äussere Parenchymzone schmäler, die Längsmuskeln sind in Folge dessen bedeutend weniger zahlreich. Wie bei Iania depressa, Dav. tetragona, Dav. tauricollis, Dav. musculosa n. sp. gehen am Hinterrande jeder Proglottis, da wo die Längsinuskulatur die subcuticularen Zellen fast berührt, zahlreiche Längsfasern in's äussere Parenchym und fixiren sich an der Cuticula. Die Längsmuskeln, namentlich die innersten, sind sehr stark und besitzen einen grossen, sich wenig färbenden Myoblasten. Die Transversalmuskulatur ist deutlich entwickelt und ihre feinen Fasern strahlen lateral in das Rindenparenchym aus. Die Dorsoventralmuskulatur ist namentlich an den seitlichen Partien der Proglottis ausserhalb des Wassergefässes als dichtes System von feinen Fasern sichtbar.

Das Parenchym ist ein feinmaschiges Gewebe, das namentlich ausserhalb der Transversalmuskulatur zwischen den Längsmuskeln erfüllt ist von zahlreichen Kalkkörperchen (Durchmesser $0,013 \mathrm{~mm}$.). Diese sind von kugeliger Gestalt, enthalten einen dunklen Kern, welcher von einer oft durch die Wirkung der Reagentien eingedrückten, sich ebenfalls dunkel färbenden Membran umschlossen ist. Nach hinten nimmt die Zahl der Kalkkörperchen im Rindenparenchym bedeutend ab und sie treten dann in den Eikapeln (s. d.) auf.

Das Excretionssystem besteht aus 4 Längsstämmen, von welchen aber nur der ventrale bis ganz nach hinten geht, um dort bei fehlendem Endglied getrennt auszumünden. Das ven- 
traie Gefäss ist überaus mächtig, auf dem Querschnitt länglich oval, dorsal und ventral die Transversalmuskulatur fast berührend. In den reifen Gliedern misst das Wassergefäss im Verticaldurchmesser 0,27 mm., in der Queraxe 0,08 mm. Das verbindende Quergefäss am Hinterende jeder Proglottis ist ebenfalls sehr weit.

Das dorsale Gefäss ist bedeutend englumiger und kreisrund im Querschnitt (Durchmesser ca. 0,013 mm.). Seine Umhüllung ist im Gegensatz zu der des ventralen Gefässes eine starke, sich dunkelfärbende Membran, welche dicht bedeckt ist von dunkelkernigen Zellen von anderer Struktur als diejenigen des Parenchyms. Das dorsale Wassergefäss geht bis weit nach hinten und wird wohl die Funktion der Ausscheidung und Aufnahme der Excretionsprodukte übernehmen, während das ventrale mehr die Ableitung der Stoffwechselprodukte zu besorgen hat.

Das Nervensystem besteht aus zwei ausserhalb der Längsgefässe verlaufenden mächtigen Nervenstämmen.

Der männliche Geschlechtsapparat setzt sich zusammen aus Cirrusbeutel mit Cirrus, Vas deferens, Vasa efferentia und Hodenbläschen. Der Cirrus mündet mit der Vagina immer auf derselben Seite in der Mitte des Proglottidenrandes in die wenig tiefe Genitalkloake aus. Der Cirrusbeutel ist kurz und reicht nur bis in die Nähe des ventralen Wassergefässes, wobei das Vas deferens zwischen ventralem und dorsalem Excretionsstamm durchlaufend in den Cirrusbeutel mündet. Der Cirrusbeutel besteht ausschliesslich aus einer überaus starken Ringmuskulatur, die hinten am mächtigsten ist. Ihre Mächtigkeit beträgt im Maximum 0,016 mm. bei einer Länge von 0,18 mm. und einem Durchmesser von 0,062 mm. des Cirrusbeutels. In diese Penistasche, deren innerer Hohlraum in der Mitte durch eine nach innen vorspringende Verstärkung ihrer muskulösen Wandung leicht eingeschnürt und von zartem Parenchym erfüllt ist, 
mündet das Vas deferens, die starke Muskulatur in Begleitung der Prostatazellen durchsetzend, welch' letzteres namentlich deutlich an den jüngeren Proglottiden zu sehen ist. Innerhalb des Cirrusbeutels erweitert sich der Samenleiter zu einer kleinen, spindelförmigen, von Diagonalmuskeln umhüllten Samenblase, welche das Sperma in den mächtigen, von einem feinen Canal, dem eigentlichen Samenleiter $(0,0027 \mathrm{~mm}$.), durchzogenen Cirrus presst. Der Cirrus besitzt einen Durchmesser von 0,019 mm. und ist unbewaffnet. Das Vas deferens schwillt kurz nach seinem Austritt aus dem Cirrusbeutel zu einer kleinen, von Prostatazellen bedeckten Vesicula seminalis an, die wie bei Dav. circumvallata (CRETY 1890) nur in den Proglottiden, welche in vollster Geschlechtstätigkeit sind, deutlich sichtbar ist. Es verläuft in unregelmässigen Spiralen zur Mitte der Proglottis und ist auf seinem ganzen Verlaufe umhüllt von grossen Prostatazellen. In der Mitte jedes Gliedes am Hinterrande löst es sich in die unter sich anastomosirenden Vasa efferentia auf, die zu den beiderseits der weiblichen Geschlechtsorgane liegenden Hodenbläschen führen. Die Hodenbläschen liegen in doppelter Lage dicht beisammen, ca. 60 an der Zahl. Die sie umhüllenden Membranen berühren sich oft und ist in Folge dessen ihre äussere Form häufig eine unregelmässige.

Der weibliche Geschlechtsapparat. Die Vagina mündet hinter dem Cirrus in die Genitalkloake; sie ist in ihrem Anfangsteil sehr dickwandig und von einem muskulösen Vaginabeutel umhüllt. Auf dem Querschnitt kann man von aussen nach innen folgende Schichten unterscheiden : eine äusserste Schicht dichteren Parenchyms, welches vielleicht auch Drüsenzellen enthält, darauf folgen spärliche Längs- und zahlreichere Ringmuskelfasern, welche der Vaginawandung direct anliegen. Diese zeigt eine äussere, mit Hämalaun nur wenig färbbare Zone und eine dickere dunkelgefärbte innere, welche das Aussehen von verklebten Cilien hat. Hinter diesem Vaginabeutel verengt sich 
die Vagina plötzlich zu einem äusserst feinen Kanal, welcher das Zurückströmen des Spermas verhindert. Von diesem Verschlussapparat an, den ich ebenfalls bei $T$. depressa, $T$. dujardinii, Dav. tauricollis und Dav. musculosan. sp. gefunden, ist die Vagina ein von zarter Membran umgebener, leicht gewundener Kanal, der in der Nähe des Keimstockes zu einem schwachen Receptaculum seminis anschwillt. Zur Zeit der grössten Geschlechtstätigkeit funktionirt die ganze Vagina von ihrer Verengerung bis zum Keimstock als Samenbehälter. Vom Receptaculum seminis an sind die weiblichen Geschlechtsgänge von einem deutlichen Epithel ausgekleidet, welchem aussen dunkel gefärbte Kerne des Parenchyms anliegen. Diese Epithelzellen, die übrigens in den ganz jungen Gliedern die ganze Vagina auskleiden, schwinden ebenfalls da wo der Oviduct in den Keimstock mündet, dafür ist hier die Wandung bedeutend stärker, und wie mir scheinen will, von feinen Fibrillen bekleidet. Der Keimstock zeigt in seinem Baue auffallende Aehnlichkeit mit demjenigen von Dav. struthionis Houttyn (Linstow 1894) indem die Eier ebenfalls zu mehreren vereinigt im Parenchym liegen. Bei Durchsicht der Flächenschnitte schien es als ob wie bei der von Linstow beschriebenen Davainea, vom Receptaculum seminis aus Aeste zu den Eiballen abgehen, welche den Samen zu sämmtlichen Einzelovarien leiten. Nach genauerem Studium dieser Verhältnisse auf Quer- und Sagittalschnitten stellte es sich heraus, dass ganz andere Verhältnisse vorlagen, Verhältnisse wie ich sie auch bei Dav. tauricollis und Dav. musculosa mit aller Sicherheit nachweisen konnte.

Leider bestand das mir zur Verfügung stehende Material von Davainea struthionis nur aus noch nicht geschlechtlich reifen Individuen. Es zeigten die sehr gut conservirten Scolices ein sehr deutliches Rostellum von 0,42 mm. Länge und einem Durchmesser von $0,68 \mathrm{~mm}$., und nicht wie Linstow angiebt, nur eine flach vorgewölbte Scheitelgegend. Bewaffnet ist das- 
selbe mit einem doppelten Kranz der von Linstow genauer beschriebenen typischen Hacken mit eigentümlich aufgefasertem Hebelast.

Die Eizellen von Dav. leptosoma sind 0,019 $\mathrm{mm}$. gross und besitzen einen grossen, sehr wenig färbbaren Kern mit dunklem Nucleolus; das umgebende Plasma besitzt ebenfalls sehr wenig Aufnahmevermögen für Farbstoffe und ist sehr vacuolenreich.

Der Dotterstock am Hinterrande der Proglottis ist ganz ventral in der Mitte des Gliedes gelegen und zeigt schwach eingebuchtete Umrisse.

Die Zusammenmündung der einzelnen Organe ist überaus verwickelt. Von der Vereinigungsstelle der einzelnen den Keimstock darstellenden Eituben verläuft der Oviduct zuerst ventral bis in die Nähe der Transversalmuskulatur, wo er nach oben umwendet und in der Mitte der Höhenausdehnung des Gliedes einerseits in die Vagina sich fortsetzt, anderseits in einen weiter dorsal gelegenen Ootyp, der von einem Schalendrüsenkomplex umhüllt ist und in dessen Nähe auch der direct dorsal aufsteigende Dottergang einmündet. Vom Ootyp führt ein als Eileiter funktionirender Gang nach dem Vorderrand der Proglottis, wo er in den Uterus einmündet und zwar in derselben Höhe in der der Oviduct aus dem Keimstock ausmündet, so dass in Folge der Kürze der Proglottiden leicht Verhältnisse vorgetäuscht werden, wie sie DIAMARE (1893) bei Dav. tetragona beschrieben hat.

Der Uterus liegt am Vorderrand der Proglottis und sendet von hier aus Schläuche ventralwärts und zwischen die Hodenbläschen; doch bald verschwindet seine Wandung und die Eier werden einzeln vom Parenchym eng umschlossen. Das umgebende Parenchym macht nun eine Reihe eigentümlicher Veränderungen durch bis die definitiven, bis ca. 20 Eier umfassenden Eikapsein gebildet sind. Die Eier, von einer enganliegenden kaum sichtbaren Membran umgeben, liegen wie schon bemerkt 
einzeln im Parenchym, das noch kurze Zeit sein normales Aussehen bewahrt. Es treten dann in der Nähe der Eier mit Hämalaun sich dunkel färbende Körner auf, die gruppenweise beisammen liegen und zu wachsen scheinen, bis sie in der werdenden Eicyște grossenteils das Aussehen von Kalkkörperchen angenommen haben, welch' letztere im äusseren Parenchym an Zahl abnehmen, sich also wohl aufgelöst und in das veränderte, die Eier umgebende Parenchym gewandert sind. Es beginnt sich bald ein freier Raum um das Ei zu bilden, zugleich verändert sich das Parenchym, es wird sehr feinmaschig und erscheint wohl desshalb dunkler gefärbt; ungefähr gleichzeitig bildet sich eine zweite zarte Schale um das Ei. Das feinmaschige Parenchym, erfüllt von Körnchengruppen verschiedener Grösse, umfasst eine grössere Zahl von Eiern (bis ca. 20). Die Hodenbläschen, das Vas deferens, die Vagina und Reste des Dotterstockes finden sich zusammengepresst und degenerirt in den noch unveränderten Resten des Parenchyms liegend, zwischen den die ganze Höhe der Proglottis einnehmenden Eicysten. In der Rindenparenchymschicht bleiben der Cirrusbeutel und der muskulöse Anfangsteil der Vagina erhalten.

Das zwischen den Eicysten liegende, noch unveränderte Parenchym verändert sich indem es überaus weitmaschig wird und somit ist erst mit Veränderung des ganzen inneren Parenchyms die Bildung der einen eigentümlichen Anblick gewährenden Eibehälter beendigt (Fig. 2).

\section{Davainea tauricollis.}

(Chapmania tauricollis Cнарм. ; Tænia argentina Zscнокке.)

(Fig. 3-כั.)

ZsCHUKKE 1888, 1895.

Diese interessante Tænie aus Rhea americana ist von ZscHoKKE 1888 beschrieben worden und hat derselbe später (1895) an sehr gut conservirtem Material den Scolex einer 
Nachuntersuchung unterworfen und dabei gefunden, dass äusserst zahlreiche stark gekrümmte Häckchen die Seitenwandungen des als Saugnapf erscheinenden, stark zurückgezogenen Rostellums bedecken und dass ferner das in das «Saugnapflumen » vorspringende Polster von zwei Hackenreihen(ZscHoKKE 1895, pag. 637) umkränzt ist. Ich konnte diesen Cestoden ebenfalls einer genaueren Untersuchung unterwerfen und kann das in ZschоKKE's neuester Arbeit hierüber gesagte nur bestätigen und zugleich einiges nicht unwesentliche beifügen.

Die Hacken des Rostellums sind, wie aus Fig. 3 ersichtlich, typische, 0,008 mm. grosse Davaineenhacken, in ihrer Form denjenigen von Davainea tetragona (BLANCHARD 1891, Fig. $15 \mathrm{C})$ sehr ähnlich. Ausser am Rostellum fand ich an einem Saugnapf Reste einer Hackenbewaffnung.

Fragen wir uns nun ob diesem typischen Davainea-Scolex nicht eine ebensolche Organisation der Geschlechtsorgane entspricht, so muss ich dies nach meiner eingehenden Untersuchung vollständig bejahen.

Die Geschlechtsorgane sind schon sehr früh angelegt und zwar treten die weiblichen und männlichen fast gleichzeitig auf. Die Hoden liegen auf einem Stadium das bereits alle Organe angelegt zeigt, am ganzen Hinterrande der Proglottis, hauptsächlich aber dicht zusammengedrängt an den seitlichen Randpartien der inneren Parenchym-Schicht. Der Cirrus besteht aus einer schlauchförmigen Zellanhäufung, die bis in die Mitte des Gliedes reicht und dort die Keimdotterstockanlage berührt. Weder das Vas deferens noch die Vasa efferentia sind auf dieser Entwicklungsstufe angelegt. Die weiblichen Geschlechtsdrüsen bestehen aus einer noch vereinigten sehr kleinen Zellmasse, welche in der Witte der Strobila liegt und von welcher ein schmaler Zellstrang, die Vagina, zu ihrem Rande verläuft; über dem Wassergefässstamm verschmilzt sie mit der Anlage des Cirrusbeutels uıd führt vereinigt mit dieser zu der bereits 
ziemlich tief eingestülpten Cuticula, der Anlage der Genitalkloake.

Im vollkommen entwickelten Zustande zeigen die Geschlechtsorgane nicht mehr die deutliche Anordnung in eine vordere, weibliche und eine hintere, männliche Zone, indem der Dotterstock nun den Hinterrand der Proglottis berührt.

Die Geschlechtskloake ist lang, schmal und nach hinten trichterförmig verengert. Sie ist von der Cuticula ausgekleidet, welcher nach innen die subcuticulare Ring - und Längsmuskulatur mitsammt den subcuticularenZellen anliegt. Bei einer Breite der Strobila von 2,2 mm. und einer Länge von $0,11 \mathrm{~mm}$. ist die Genitalkloake 0,27 mm. tief und ihr Durchmesser am hinteren Ende 0,019 mm. Der Cirrusbeutel ist bei derselben Proglottis $0,55 \mathrm{~mm}$. lang mit einem Durchmesser von 0,11 mm. Er ist hauptsächlich aus Ringmuskeln gebildet, welchen aussen wenige Längsmuskeln anliegen. Die Ringmuskulatur ist im hinteren Teil bald mehr, bald weniger mächtig, so dass das innere, mit zartem Bindegewebe erfüllte Lumen des Beutels in ungleich grosse Kammern unvollkommen getrennt erscheint. Im Vorderteil ist der innere Raum sehr eng, die Ringmuskulatur dagegen überaus mächtig. Wie bei allen von mir bis jetzt untersuchten Tænien mit langer Cirrustasche, besitzt dieselbe auch hier einen deutlichen Retraktor Penis, der in die Transversalmuskulatur übergeht. Die Wandung des Cirrus ist stark und gleich structurirt wie die die Genitalkloake auskleidende Cuticula, so dass sie gleichsam in diese überzugehen scheint, obwohl die Entstehung des Penis deutlich das Gegenteil zeigt. Der Cirrus besitzt denselben Durchmesser wie der Grund der Genitalkloake, so dass er in erigirtem Zustande die Vagina vollkommen abschliesst, ähnlich wie bei Tcenia depressa (s. I. Beitrag 1895, pag. 454). Er ist am Vorderende mit feinen Borsten besetzt.

Das Vas deferens, von grossen Prostatazellen umhüllt, macht überaus zahlreiche Windungen, die zum grössten Teil ventral- 
wärts vom Cirrusbeutel verlaufen. Eine Vesicula seminalis fehlt.

Der mächtige Cirrus und die zahlreichen Windungen des Vas deferens nehmen fast die ganze linke Hälfte der Proglottis ein, so dass nur ca 20 Hodenbläschen auf dieser Seite dicht zusammengedrängt Platz finden, während auf der gegenüberliegenden Seite etwa 60 derselben liegen. Die Hodenbläschen sind ziemlich gross, oval, ihr grösster Durchmesser, in der Höhenrichtung der Proglottis liegend, beträgt $0,10 \mathrm{~mm}$.

Die weiblichen Geschlechtsdrüsen sind verhältnismässig gross und liegen in der Mitte des Gliedes. Der Keimstock erscheint äusserlich als fast einheitliche Zellmasse, in welcher aber deutlich eine gruppenweise Vereinigung der Eizellen zu sehen ist. Die Eizellgruppen scheinen von einer überaus zarten Membran umgeben zu sein. Die Eizellen selbst sind 0,023 mm. gross mit hellem grossem Kern, der ein feines Plasmagerüst und einen sich dunkel färbenden Nncleolus enthält. Der Keimstock breitet sich nach allen drei Richtungen des Raumes so stark aus, als die männlichen Geschlechtsorgane es erlauben. Nicht so der Dotterstock, der auf Flächenschnitten, sehr schmal, den Hinterrand der Proglottis einnimmt, auf Querschnitten dagegen eine bedeutende Mächtigkeit zeigt. Seine Zellen bestehen aus einem grobkörnigen Plasma, das einen ebensolchen Kern umschliesst. Die Zusammenmündung der Organe und den Verlauf der Geschlechtsgänge konnte ich, ohne zur leicht irreführenden Reconstruction greifen zu müssen, an einem überaus günstigen Schnitt (Fig. 4) deutlich erkennen. Der dem Keimstock entspringende Oviduct ist in seinem Anfangsteil von zarten Fibrillen bedeckt und wie die übrigen weiblichen Geschlechtsgänge, mit Ausnahme der Vagina, von einem Epithel ausgekleidet dem aussen Zellkerne anliegen. Dieser Oviduct. nachdem er ventralwärts verlaufend die Transversalmuskulatur erreicht hat, wendet sich nach der Dorsalfläche um, wo er sich in der Mitte der Höhe des Gliedes einerseits in die Vagina, andererseits in einen weiter dorsal stre- 
benden Gang fortsetzt. Dieser letztere mündet unterhalb der dorsalen Transversalmuskulatur in den Ootyp. Die Wandung des Ootyp ist ebenfalls von Epithel gebildet und wird von den zahlreichen Schalendrüsenzellen durchbrochen. In ihn mündet der enge Dottergang ein und geht der Eileiter ab, der ventralwärts die befruchteten und mit Dotter und Schalendrüsenmaterial versehenen Eier in den Uterus fuhrt. Es ist also auch hier, wie bei Dav. leptosoma, ein Uterus vorhanden und mündet der Oviduct nicht in den Keimstock, wie dies nach Diamare (1893) bei Dav. tetragona der Fall.

Die Vagina schwillt nicht weit von ihrer Teilungsstelle zu einem langgestreckten Receptaculum seminis an. Da aber ein Verschlussapparat vorhanden, funktionirt die ganze Vagina bis zu diesem als solches. Von der das Zurückströmen des Spermas verhindernden plötzlichen Verengerung der Vagina bis zur Genitalkloake ist dieselbe dickwandig und von feinen Längsfasern bedeckt, welchen aussen dichteres Parenchym und Drüsenzellen anliegen. Der Verlauf der Vagina ist, wie bereits ZsCHOKKE festgestellt, vollkommen dorsal und ist diese also über dem Cirrus gelegen.

Der Uterus liegt in seinem ersten Entwicklungsstadium vor dem Keimstock und sendet von hier seine Fortsätze ventralwärts, von wo aus sie sich dorsalwärts ausdehnen und so die Geschlechtsorgane verdrängen, bis schliesslich nur noch ein eigentümliches, noch zu beschreibendes Organ, ein kurzes Stück des Vas deferens, der veränderte Cirrusbeutel und Reste der Tagina übrig bleiben. Anfangs erscheint der Uterus gekammert; die einzelnen Abteilungen sind von sich dunkler färbenden Parenchymzellen umgeben, welche bald zwischen die Eizellen wuchern und jedes Ei einzeln mit einer spärlichen Bindegewebehülle umgeben (Fig. 5). Die Eier (0,033 mm.) sind von zarter Membran umhüllt, welche von den drei paarigen, 0,021 mm. grossen Embryonalhacken durchbrochen wird; auf 
diese folgt eine äussere dickere Schale, deren Durchmesser 0,044 mm. beträgt.

Wie soeben erwähnt, tritt in der Mitte der reifen Proglottiden ein merkwürdiges Organ auf, das ich anfangs für die Vesicula seminalis hielt und das ZscHoKKe (1888) als Hoden beschreibt; sein sehr spätes Auftreten und seine histologische Structur sprechen entschieden gegen diese Auffassung, vor allem aber der Umstand, dass sich in jedem Gliede ca. 80 Hodenbläschen finden. Dieses eigentümliche Gebilde, über dessen Funktion ich vollkommen im Unklaren bin, zeigt anfangs eine kugelige Form, die später sich in der Längsrichtuug der Proglottis streckt und eine starke Eiuschnürung erhält, so dass dieses die ganze dorsoventrale Mächtigkeit des Parenchyms einnehmende Organ in einen vorderen grösseren und einen hinteren kleineren Teil zerfällt. Seine Umgrenzung ist nicht scharf, sondern geht in das umliegende Parenchym über; es ist namentlich in seiner hinteren Partie umgeben von Kalkkörperchen, enthält aber selbst auch solche. Die Structur des Ganzen ist die des Parenchyms, nur viel dichter als dieses und erscheint das Gebilde desshalb dunkler gefärbt.

\section{Davainea (?) musculosa nov. spec.}

(Fig. 6-9.)

Dieser neue Cestode aus dem Darme von Sturnus vulgaris gehört in die nur wenige Arten umfassende Gruppe von Davaineen mit alternierender Ausmündung der Geschlechtsorgane. Seine Länge beträgt ca. $6 \mathrm{~cm}$. und seine grösste Breite 1,3 mm. Der Scolex (Fig. 6) ist nicht deutlich von der Strobila abgesetzt, sondern geht allmählig in diese über. Da wo der Halsteil die Breite der Proglottidenkette erreicht hat, beginnt auch die Strobilation. Der Kopf zeigt einen Durchmesser von $0,27 \mathrm{~mm}$. und ist mit 4 verhältnissmässig grossen Saugnäpfen und einem in der äusseren Form dem von Dav. struthionis (s. d. 
Arbeit pg. 115) ähnlichen Rostellum bewaffnet. Das Rostellum misst $0,085 \mathrm{~mm}$. im Durchmesser und seine Länge beträgt 0,06 mm. In Folge leichter Maceration fehlten sowohl dem Rostellum, als den Saugnäpfen die Hacken, so dass sich die Einreihung dieser neuen Art in das Sub-Genus Davainea nur auf den Bau des Keimstockes, den Verlauf der Geschlechtsgänge und die Anwesenheit von Eicysten basirt. Gegen diese Unterbringung spricht einzig der Bau des Rostellums, das aus zwei ineinander geschachtelten Muskelsäcken besteht. Es ist aber unsere Kenntnis der Structur des Rostellums noch zu mangelhaft und eigentlich nur bei Davainea tauricollis und contorta (ZscHOKKE 1888 und 1895) genauer untersucht, um hierauf besonderes Gewicht legen zu können. Die äussere Gestalt der Proglottiden ist in Folge der sehr differencirten Muskulatur innerhalb derselben Strobilenkette ziemlich variabel, so dass ich auf Massangaben verzichte und auf Figur 7 verweise, welche die Durchschnittsform der Proglottiden wiedergiebt.

Die Körperdecke besteht aus einer sehr starken $(0,013 \mathrm{~mm}$.) Cuticula unter welcher ein System von Ring- und Längsmuskeln liegt. Die subcuticularen Zellen sind $0,06 \mathrm{~mm}$. lang und gestreckt keulenförmig.

Das Parenchym zeigt keine besonderen Eigentümlichkeiten; es enthält in seiner sog. Rindenschicht spärliche Kalkkörper eingestreut.

Die Muskulatur ist nicht nur stark entwickelt, sondern auch in ihrem Verlaufe mannigfaltig differencirt. Die Längsmuskulatur ist in Bündeln von bis 14 Fasern zusammengefasst und zwar in eineu äusseren und einen inneren Ring von solchen. Sie trennt das Bindegewebe in eine äussere und eine innere Parenchymschicht. Da die Einschnürungen der Strobilenketten so stark sind, dass sie die Längsmuskulatur berühren, so ist das Rindenparenchym jedes Gliedes fast ohne Verbindung mit demjenigen 
der nächstfolgenden und der vorausgehenden Proglottis. Von der Einschnürungsstelle strahlen zahlreiche Längsfasern nach vorn in's äussere Parenchym aus, heften sich an der Cuticula an und tragen so zu der grossen Formveränderlichkeit der Strobilen bei. Innerhalb des inneren Längsmuskelmantels verläuft die überaus schwache Transversalmuskulatur; dieselbe zeigt an der Grenze je zweier Proglottiden eine sehr bedeutende Verstärkung, wie ich solche ausser bei den im ersten Beitrag beschriebenen Taenia depressa und capitellata, auch bei $T$. serpentulus Schrank, T. constricta Molin, T. puncta von Linstow und bei einer unbestimmbaren interessanten Vogeltænie aus Tardon vulpanser vorgefunden habe. In etwas anderer Art findet sich dieselbe Vorrichtung bei der Amphibientænie $T$. dispar Gœe wo nicht ein Ringmuskel sondern ein ganzes Netz von Muskeln an der Uebergangsstelle der sich einzeln lostrennenden Proglottiden findet (Fuhrmann 1895, pg. 211). Dieser bis jetzt merkwürdigerweise übersehene Muskelring ist oft deutlich in zwei Ringe geteilt, einen für das Hinterende des vorderen Gliedes, den zweiten für den vorderen Teil der folgenden Proglottis. Ueber die mutmassliche Funktion dieser Vorrichtung habe ich mich im ersten Beitrag dahin ausgesprochen, dass dieselbe wohl einesteils zur Fortbewegung der Kette und Abschnürung der Proglottiden, ferner auch zum Verschlusse der durch die Ablösung des Gliedes entstehenden Wunde dienen mag.

Die Dorsoventralmuskulatur ist besonders deutlich am Uebergange je zweier Glieder entwickelt und wird dort die Funktionen der Ringmuskeln unterstützen. Die feinen Fasern zeigen einen deutlichen Myoblasten.

Das Wassergefässsystem besteht aus zwei Paaren von Längsstämmen von welchen der ventrale ca. 0,03 mm., der dorsale, nur wenig weiter nach innen gelegene, $0,0056 \mathrm{~mm}$. Durchmesser besitzt. Das dorsale Gefäss ist sehr dickwandig von einem zarten Plasmamantel mit kleinen Kernen umgeben, 
das ventrale Gefäss ist zartwandig und sendet zahlreiche Seitenstämme sowohl in's äussere als in's innere Parenchym. Das ventrale Verbindungsgefäss ist etwa um die Hälfte enger als die Längsstämme.Ihre Ausmündung im Endglied erfolgt durch eine Expulsionsblase. Diese ist sehr muskulös; ihrer cuticularen Auskleidung direct anliegend findet sich zunächst eine Schicht Ringmuskeln die nicht an der Ausmündungsstelle, sondern am hinteren Ende der Blase am mächtigsten entwickelt sind; hierauf folgt eine Längsmuskelschicht. Beide Muskelsysteme sind stärker entwickelt als die ihnen entsprechende subcuticulare Muskulatur. An den Wandungen der Excretionsblase heften sich zahlreiche Muskeln an, die nach dem Hinterende des Gliedes verlaufen (Fig. 8).

Seitlich von den Gefässen durchziehen zwei Nervenstämme das Parenchym, welche im Scolex durch eine Commissur mit einander verbunden sind.

Die Geschlechtsorgane zeigen in ihrer Entwicklung keine Besonderheiten. Sie sind in geringer Entfernung vom Scolex bereits vollständig ausgebildet. Die beiden Geschlechtsgänge münden in eine wenig tiefe Genitalkloake, welche, unregelmässig abwechselnd (bald rechts bald links), sehr nahe dem Vorderrand der Proglottis liegt. Cirrus und Vagina, letztere hinter ersterem, verlaufen zwischen dem ventralen und dem dorsalen Längsgefäss des Excretionssystems durch und biegen je nach dem Contractionszustand des Gliedes mehr oder weniger stark nach der sehr weit nach vorn verschobenen Geschlechtskloake um (Fig. 7). Der Cirrusbeutel ist $0,19 \mathrm{~mm}$. lang und besitzt einen Durchmesser von $0,022 \mathrm{~mm}$.; ich habe an demselben nur Längsmuskeln unterscheiden können, die sich am Hinterende in einen langen Retractor Penis fortsetzen. Der Cirrus ist fein bedornt und das Vas deferens in mehreren Schlingen im hinteren Teil der Penistasche aufgewickelt. An mehreren aufeinander folgenden Gliedern konnte ich sehen wie der Penis in die enge, 
durch ihn erweiterte Vagina eingeführt war. Das aus dem Cirrusbeutel austretende Vas deferens ist mehrfach gewunden und es fehlt eine Vesicula seminalis, wie bei fast allen bis jetzt bekannten Davinea-Arten, so auch hier. Der Samenleiter löst sich in der Mitte der Proglottis in die leicht sichtbaren Vasa efferentia auf, die dorsal in die Hodenbläschen einmünden. Die Hoden finden sich in der hinteren Hälfte der Proglottis in der Zahl von ca. 25 ; sie nehmen einzeln die ganze Höhe der Proglottis ein und liegen ziemlich dicht gedrängt nebeneinander.

Die weiblichen Geschlechtsorgane erfüllen den ganzen vorderen Teil der Proglottis. Die Vagina ist in ihrem Anfangsteil sehr dickwandig, von feiner Ringmuskulatur umgeben, der aussen ein vom umgebenden Parenchym sich deutlich abhebender Zellenbelag aufliegt.

Nach einem Verlaufe von etwa 0,28 mm. verengert sich die Vagina plötzlich zu einem äusserst feinen $0,027 \mathrm{~mm}$. langen Kanälchen, das sich zu dem von feiner Membran umhüllten Receptaculum seminis erweitert. Der weitere Verlauf der Geschlechtsgänge ist schwierig festzustellen, doch scheinen sie mir durchaus denselben Weg einzuschlagen wie bei Dav. tauricollis und leptosoma. Es teilt sich die Vagina in einen absteigenden Ast der zum Ovarium führt und einen aufsteigenden, der an seinem dorsalsten Punkte die Schalendrüsengruppe durchquert und den Dottergang aufnimmt, worauf er ventralwärts in den Uterus führt.

Der Keimstock zeigt denselben äusseren Bau wie bei anderen Tænien, es liegen aber die Eier zu Gruppen vereinigt dicht beisammen. Der Dotterstock, am meisten ventral gelegen, ist gross und deutlich gelappt. Die Schalendrüsen sind von allen weiblichen Organen am meisten dorsal gelegen.

Der Uterus sendet von einem Punkte aus strahlenförmig Eischläuche in's Parenchym, die ihre Wandung bald verlieren, so dass die Eier in's Bindegewebe zu liegen kommen, wo sie 
dann von besonders differencirten Parenchymzellen umwuchert werden. Auf dem Querschnitt durchziehen die Eier in einfacher Lage wellenförmig die Proglottis, bis ins äussere Parenchym dringend; sie sind dicht umhüllt von dunkelgefärbten Parenchymzellen und selten mehr als zu 2 oder 3 vereinigt. Auf dem Flächenschnitt ist, nachdem die Uteruswände verschwunden, die Anordnung der Eier eine durchaus unregelmässige. Die Eier sind 0,018 mm. gross, von einer eng anliegenden Schale umgeben. Die Bildung der Eikapseln scheint auch in den letzten Gliedern der mir zur Verfügung stehenden Exemplare nicht vollkommen beendet zu sein.

\section{ALLGEMEINE BEMERKUNGEN}

über das Sub-Genus Davainea.

Seit Blanchard (1891), der eine Zusammenstellung aller bekannten Vertreter des Sub-Genus Davainea gegeben, hat sich die Zahl derselben fast um die Hälfte vermehrt, so dass es sich verlohnt, die Liste zu ergänzen.

Nach Blanchard teile ich die Davaineen ein in solche mit abwechselnder und solche mit einseitiger Ausmündung der Geschlechtsgänge.

a) Mit alternirenden Geschlechtsöffnungen :

1. Davainea proglottina Davaine aus Gallus domesticus.

2. Davainea echinobothrida Megnin aus Gallus, Phasianus und Columba.

3. Davainea circumvallata Krabbe, identisch mit T. pluriuncinata Crety aus Coturnix communis.

4. Davainea cesticillus Molin aus Gallus und Phasianus.

*5. Davainea spinosissima Linstow aus Turdus merula.

*6. Davainea musculosa nov. spec. aus Sturnus vulgaris.

*7. Davainea rectractilis Stiles aus Lepus arizona. 
b) Mit einseitigen Geschlechtsöffnungen :

8. Davainea insignis Steudener aus Carpophaga oceanica.

9. Davainea australis Krabbe aus Dromaus nove Hollandice.

10. Davainea urogalli Modeer aus Tetrao tetrix, T. urogallus, Megaloperdix nigelli und Perdix graca.

11. Davainea frontina Duj. aus Oviolus galbula, Picus viridis und Picus major.

12. Davainea tetragona Molin, identisch mit Tania bothroplitis Piana aus Gallus.

13. Davainea columba Zeder, identisch mit Tania crassula Rud. aus Columba livia und C. turtus.

14. Davainea circumcincta Krabbe aus Ardea garezetta.

15. Davainea Friedbergeri v. Linstow aus Phasianus colchicus.

16. Davainea leptosoma Diesing aus Psittacus.

*17. Davainea struthionis Houttoyn aus Struthio camelus,

* 18. Davainea tauricollis Chapm., identisch mit Chapmania tauricollis Chapm. und Tania argentina Zschokke aus Rhea americana.

19. Davainea (?) contaniana Polonia aus Meleagris gallopado.

*20. Davainea (?) clavulus v. Linstow aus Ptilostris Alberti.

21. Davainea madagascariensis Davaine aus Homo.

22. Davainea contorta Zschokke aus Manis pentadactyla.

*23. Davainea salmonis Stiles aus Lepus melanotis und L. sylvaticus.

Von diesen 23 Vertretern des Genus Davainea sind nur 10 anatomisch genauer untersucht und zwar :

Dav. proglottina Dav. (BlanchaRd 1891), Dav. urogalli Modeer (Morell 1895), Dav. circumvallata Krabbe (Crety 1890), Dav. struthionis Houttoyn (v. Linstow 1894), Dav. tetragona Molin (Filippi 1892, Diamare 1893), Dav. madagascariensis

* Alle mit einem Sternchen versehenen Arten sind zum Verzeichnis BLANCHARD's neu hinzugekommen. 
Dav. (Leuckart 1891), Dav. contorta Zschokke (ZschoKKe 1895), Dav. tauricollis Chapm. (ZsсHоKKE 1888, 1895 und diese Arbeit, pag. 117, Dav. musculosa nov. spec. (s. diese Arbeit, pag. 123), Dav. leptosoma Dies (s. diese Arbeit, pag. 111).

Von diesen bedürfen die vier ersten einer Nachuntersuchung, hauptsächlich desshalb, weil den Autoren keine vollkommen reifen Proglottiden zur Verfügung standen, anderseits auch weil die Zusammenmündung der Geschlechtsorgane nicht vollkommen klargelegt ist.

Die von BLANCHARD 1891 gegebene Diagnose ist noch heute in allen ihren angegebenen Charakteren gültig ', nur hat es sich gezeigt, dass wenn die Angaben der verschiedenen Bearbeiter von Vertretern dieses Sub-Genus richtig sind, innerhalb der Gruppe der Davaineen grosse anatomische Verschiedenheiten herrschen.

Der männliche Apparat ist überall ungefähr gleich gebaut und zeigt das gemeinsame Merkmal, dass an Stelle einer Ves. seminalis zahlreiche Windungen der Vas deferens treten, oder wenn ein solches vorhanden (Dav. circumvallata,Dav. leptosoma), dasselbe sehr klein und ein transitorisches Organ darstellt, das nur zur Zeit des Maximums der Geschlechtstätigkeit auftritt. Der Cirrusbeutel mit Cirrus ist sehr verschieden gebaut; ersterer hat nur bei Dav. tauricollis und musculosa wegen der Länge der Penistasche einen Retractor Penis. Der Cirrus ist bald bewaffnet, bald ohne Dornen. Die Zahl der Hoden ist mit Ausnahme von Dav. contorta, welche deren nur zwei besitzt, immer eine verhältnismässig grosse. Ihre Lagerung ist sehr verschieden; bei Dav. struthionis und proglottina nehmen sie die ganze dorsale Fläche des Markparenchyms ein; bei Dav. circumvallata, Dav. tetragona und Dav. musculosa sind die Hodenbläschen

${ }^{1}$ Linstow, 1894, glaubt auf Grund des Fehlens der Saugnapfhacken bei Dav. struthionis dieses Gattungsmerkmal streichen zn müssen ; doch ist es sehr wahrscheinlich, dass die Hacken sehr leicht ausfallen.

Rev. Suisse de Zool., T. IV. 1896. 
auf den Hinterrand der Proglottis beschränkt. Eine dritte Gruppe zeigt eine Verteilung der Hoden durch die ganze Höhe des Gliedes zu beiden Seiten der weiblichen Geschlechtsorgane, so bei Dav. tauricollis, Dav. urogalli und Dav. leptosoma; Dav. contorta besitzt, wie schon erwähnt, zwei an dem der Geschlechtsöffnung gegenüberliegenden Rande der Proglottis befindliche Hoden.

Die weiblichen Geschlechtsorgane scheinen nach der Beschreibung der Autoren sehr verschieden gebaut zu sein. Von den einzelnen Drüsen des weiblichen Genitalapparates ist es der Keimstock, der sehr verschiedene Structur zeigt. Im einfachsten Fall scheint er wie bei den meisten Tænien gebaut zu sein; dies ist der Fall bei Dav. madagascariensis, Dav. contorta, Dav. proglottina und Dav. circumvallata (ZschoKKE 1895, pag. 642). Bei einer anderen Gruppe ist das Ovarium mehr oder weniger compact, zeigt aber doch im Aufbau eine gruppenweise Zusammenfassung der einzelnen Eier, somit eine beginnende Auflösung in Einzelovarien, so bei Dav. urogalli, tauricollis und musculosa. In der dritten Gruppe, gebildet von Dav. struthionis, tetragona und leptosoma, liegen die Eier zu mehreren am blinden Ende von langen Schläuchen, die sich vereinigend in den Oviduct münden.

Der Dotterstock zeigt keine besonderen Eigentümlichkeiten. Die Schalendrüse ist mit Ausnahme von Dav.struthionis überall vorhanden, ebenso ein Ootyp; dagegen zeigt nur Dav. madagascariensis einen Schluckapparat. Aehnliche Vorrichtung, bestehend aus sehr zarter Muskelfibrillenbekleidung des Anfangsteils des Oviductes, finden sich bei Dav. tetragona, Dav.tauricollis und leptosoma.

Die Bildung der reifen Eier scheint auf sehr verschiedenem Wege vor sich gehen zu können, so dass, wenn diese Verschiedenheiten wirklich bestehen, man berechtigt wäre, das SubGenus in drei Gruppen zu trennen.

Am einfachsten gestalten sich die Verhältnisse bei Dav. madagascariensis und Dav. contorta, denn hier gelangen die Eier 
auf dem gewöhnlichen Wege in einen Uterus, der seine Wandungen bald verliert, so dass nun die Eier ins Parenchym treten können, um dort von demselben umwuchert zu werden.

Aehnliche Verhältnisse habe ich bei allen von mir untersuchten Davaineen (Dav. leptosoma, tauricollis und musculosa gefunden, so dass sich die Säugetier-Davaineen in nichts von denjenigen der Vögel unterscheiden.

In der zweiten Gruppe finden wir, dass die Eier aus dem Eierstock durch einen Oviduct zu der Schalendrüse geleitet werden, wo auch der Dotterstock einmündet; von hier, versehen mit den nötigen Materialien, kehrt die Eizelle wieder in das Ovarium zurück, welches so zum Uterus wird, der sich dann in einzelne im Parenchym zerstreute Eiballen auflöst. Diese eigentümlichen Verhältnisse hat DiamaRE (1893) bei Dav. tetragona nachgewiesen. Der Umstand, dass ich bei Dav. tauricollis anfangs den von DiamaRE angegebenen Verlauf der Geschlechtsgänge zu sehen glaubte, macht es mir als wahrscheinlich annehmbar dass auch bei Dav. tetragona Verhältnisse herrschen wie ich sie für die von mir untersuchten Davaineen gefunden; eine erneute Untersuchung dieser Art wird über die Richtigkeit dieser Vermutung entscheiden. Erst nachdem ich eine Querschnittserie zu Gesichte bekam(Fig.4), auf welcher die Anlage des Uterus eben begonnen und sich erst wenige Eier vorfanden, zeigte es sich am deutlichsten, dass ich mich getauuscht hatte, in Folge des eigentümlichen Verlaufes des Eileiters und seiner Einmündung in den Uterus, in unmittelbarer Nähe der Ausmündung des Oviductes. Es existirt bei Dav. tauricollis, wie ich dies auch mit Sicherheit für Dav. leptosoma und musculosa nachgewiesen, ein Uterus, der wie ich vermute allen Davaineen zukommt. Derselbe ist, wie bei den von LEUCKART (1892) und ZschoKKE (1895) beschriebenen Davaineen, nur von sehr kurzem Bestand, indem die Eier bald vom Parenchym umwuchert werden.

Bei der von Linstow beschriebenen Dav. struthionis bleiben 
«von Anfang bis Ende die Keimzellen in den Ovarien liegen, Samen- und Dotterzellen werden zu ihnen geleitet und die Schalensubstanz wird von den Ovarien selber abgesondert; dem entsprechend fehlt ein Ootyp, ein Schluckapparat und eine Schalendrüse.» Aehnliches will auch Morell (1895) bei Dav. urogalli gesehen haben. Bei Davainea leptosoma, deren Ovarien gleich denjenigen von Dav. struthionis gebaut sind, fanden sich bei genauer Untersuchung von Quer- und Sagittalschnitten die bereits erwähnten Verhältnisse (s. pg. 131). Ich glaube dass die sonderbaren Verhältnisse bei Dav. struthionis einer erneuten Untersuchung bedürfen, umsomehr als v. Linstow keine Proglottiden mit reifen Eiern zur Verfügung standen, somit die von ihm gemachten Angaben über die Funktion des Ovariums mehr Annahmen als wirklichen Tatsachen entsprechen mögen.

Die die Eier umhüllenden Eikapseln sind von sehr verschiedenem histologischen Bau, was schon bei Vergleich der Eihüllen der von mir beschriebenen Arten (Fig. 2, 5, 9) hervorgeht.

\section{LITTERATURVERZEICHNIS}

1869. Krabbe, H. - Bidrag til kundskab om Fuglenes Bændelorme. Vidensk. Selskabs Skrifter.

1878. Linstow, 0. v. - Compendium der Helminthologie.

1882. Krabbe, H. - Nye Bidrag til Kundskab om Fuglenes Bændelorme. Vidensk. Selskabs Skrifter.

1888. Zsснокке, F. - Ein Beitrag zur Kenntnis der Vogeltænien. Centralblatt f. Bakt. - und Parasitenkunde. Jahrg. II. (Tænia argentina Zschokke, identisch mit Chapmania tauricollis Chapm. u. Davainea tauricollis, s. d. Arbeit pg. 117.

1889. Linstow, O. v. - Nachtrag zum Compendium der Helminthologie.

1890. Crety, C. - Cestodi della Coturnix communis Bonn. Boll. dei Musei di zool. ed anat. comp. della R. Univ. di Torino. Vol. V. (Trenia (Dav.) circumvallata Krabbe, identisch mit Tænia (Dav.) pluriuncinata Crety.)

1891. Blanchard, R. - Notices helminthologiques. Bull. et mém. de la Soc. zool. de France. Vol. XVI. (Davainea proglottina Dav. ; ausserdem enthält diese Arbeit eine Zusammenstellung der Vertreter der Genera Echinocotyle, Davainea u. Ophryocotyle.) 
1891. Leuckart, R. - Ueber Tænia (Dav.) madagascariensis Dav. Verhandl. der deutschen zoolog. Ges.

1892. Filippi, C. de. - Ricerche istolog. ed anatom. sulla Tænia bothrioplitis Piana. Reale Academia dei Lincei. (Tænia bothrioplitis Piana, identisch mit Davainea tetragona Molin; die Richtigstellung der Untersuchung siehe V. Diamare 1893.)

1893. Diamare, V. - Le funzioni dell' ovario nella Davainea tetragona Molin. Rend. della R. Acad. delle sc. fis. e mat. di Napoli. (Dav. tetragona.)

1893. Linstow, 0. v. - Zur Anatomie und Entwicklungsgeschichte der Tænien. Archiv f. mikr. Anat. Bd. XLII. (Tœnia (Dav.) struthionis Houttoyn.)

1894. Linstow, 0. v. - Helminthologische Studien. Jen. Zeitsch. f. Naturw. 28. $\mathrm{Bd}$.

1894. Stiles, C.-W. - Notes sur parasites. - Bull. de la Soc, zool. de France. Vol. XIX.

1895. Moreli, A. - Anatomisch-histologische Studien an Vogeltænien. Archiv f. Naturgeschichte. (Tænia (Dav.) urogalli Modeer.)

1895. Zsснокке, F.-Davainea contortan. sp. aus Manis pentadactyla L. Centralblatt f. Bakt. und Parasitenkunde. Bd. XVII.

1895. Fuhrmann, 0. - Die Tænien der Amphibien. Zoolog. Jahrbücher Bd. 9, Abt. f. Anat.

1895. Stiles, C.-W. - Notes on Parasites; Preliminary Note to a A Revision of the adult Cestodes. The Veterinary Magazine. Vol. II.

189.5. Fuhrmann, 0. - Beitrag zur Kenntnis der Vogeltænien. Revue suisse de Zoologie. T. III. 
Tafel IV.

\section{FIGURENERKL ERUNG}

Die Figuren 3, 6, 7 und 8 sind mit dem Abbe'schen Zeichenapparat entworfen.

Davainea leptosoma. Fig. 1 und 2.

Fig. 1. Hacken des Rostellums nach Krabbe.

Fig. 2. Eikapsel. $P$, verändertes Parenchym, das zahlreiche Kalkkörperchen enthält; $H$, degenerirtes Hodenbläschen (nach einem ProglottidenFlächenschnitt gezeichnet.

\section{Davainea tauricollis. Fig. 3-丂ّ.}

Fig. 3. Hacken des Rostellums.

Fig. 4. Verlauf der weiblichen Geschlechtsgänge nach einem Querschnitt gezeichnet. $O v$, Ovarium, $D_{0}$, Dotterstock, $S d$, Schalendrüse, H, Hoden, Od. Oviduct, $R$, Receptaculum seminis der Vagina, $D g$, Dottergang, $E$, Eileiter der zum Uterus führt.

Fig. こั. Eier umhüllt von verändertem Parenchym, $P$.

\section{Davainea musculosa nov. spec. Fig. 6-9.}

Fig. 6. Scolex.

Fig. 7. Flächenansicht der Proglottis. Lm, Längsmuskulatur, Rm, Ringsmuskeln, $v W$, ventrales Excretionsgefäss mit seinen Abzweigungen $A W, d W$, dorsales Excretionsgefäss, Ci, Cirrus, $R p$, Penisretractor, Vd, Vas deferens, $H$, Hodenbläschen, $V g$, Vagina, $V a$, Verschlussapparat, Rs, Rec. seminis, $S d$, Schalendrüse, $K$, Keimstock, $D_{0}$, Dotterstock.

Fig. 8. Flächenschnitt durch das letzte Glied. $C$, Cuticulare Auskleidung der Expulsionsblase $E, R m$ und $L m$, ihre Ring- und Längsmuskulatur, $M$, Muskeln die sich einerseits an der Excretionsblase, anderseits an der Cuticula des Hinterendes der Proglottis anheften.

Fig. 9. Eier von verändertem Parenchym umgeben, das nach aussen in normales übergelıt. 


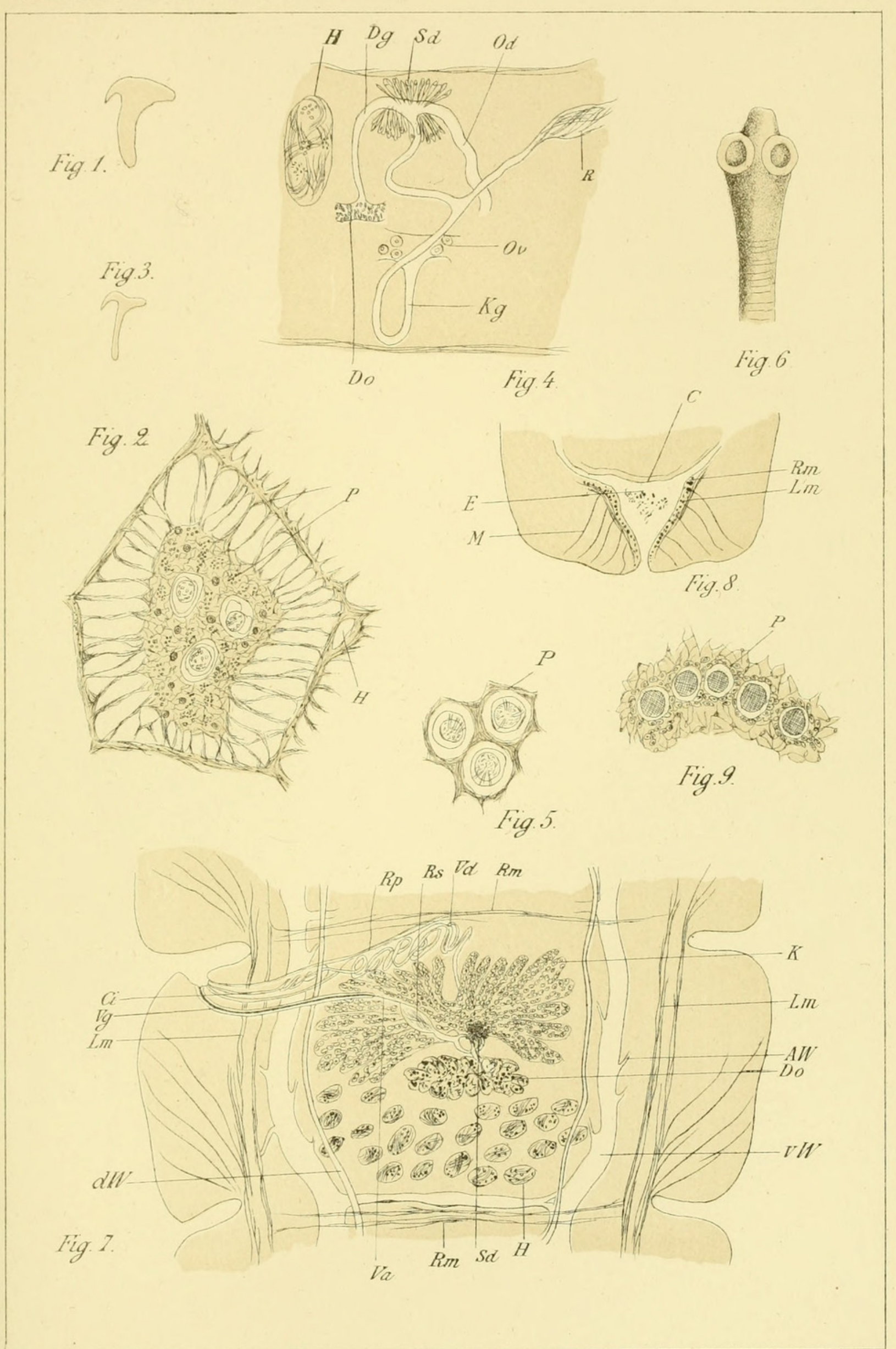

\section{O. Fuhrmann.- Vogeltaenien.}




\section{$2 \mathrm{BHL}$ Biodiversity Heritage Library}

Fuhrmann, Otto. 1896. "Beitrag zur Kenntnis der Vogeltænien." Revue suisse de zoologie 4(1), 111-133. https://doi.org/10.5962/p.318241.

View This Item Online: https://www.biodiversitylibrary.org/item/38762

DOI: https://doi.org/10.5962/p.318241

Permalink: https://www.biodiversitylibrary.org/partpdf/318241

\section{Holding Institution}

MBLWHOI Library

\section{Sponsored by}

MBLWHOI Library

\section{Copyright \& Reuse}

Copyright Status: NOT_IN_COPYRIGHT

This document was created from content at the Biodiversity Heritage Library, the world's largest open access digital library for biodiversity literature and archives. Visit BHL at https://www.biodiversitylibrary.org. 